\title{
ON A PROBABILISTIC DESCRIPTION OF SMALL SCALE STRUCTURES IN 3D FLUIDS
}

\author{
Franco FLANDOLI \\ Dipartimento di Matematica, Università di Pisa, Via Bonanno 25B, 56126 Pisa, Italy
}

Received 27 January 2000, revised 3 March 2001

ABSTRACT. - A vortex structure based on 3-dimensional Brownian motion is introduced. The interaction energy $H_{x y}$ between different vortex filaments $\left(x+W_{t}\right)$ and $\left(y+W_{t}\right)$ is rigorously defined and proved to be finite. The divergence of $H_{x y}$ as $|x-y| \rightarrow 0$ is analyzed and used to prove that the total energy of the vortex structure is finite, under suitable assumptions. A relation with the intersection local time is established. @ 2002 Éditions scientifiques et médicales Elsevier SAS

RÉSUMÉ. - Une structure tourbillonnaire basée sur le mouvement brownien tri-dimensionnel est introduite. L'énergie d'interaction $H_{x y}$ entre les différents filaments tourbillonnaires $\left(x+W_{t}\right)$ et $\left(y+W_{t}\right)$ est définie de manière rigoureuse et il est démontré qu'elle est finie. La divergence de $H_{x y}$ quand $|x-y| \rightarrow 0$ est analysée et utilisée pour montrer, sous certaines hypothèses, que l'énergie totale de la structure tourbillonnaire est finie. Une relation avec le temps local d'intersection est établie. @ 2002 Éditions scientifiques et médicales Elsevier SAS

\section{Introduction}

The geometry and statistics of small scale structures in turbulent flows is only partially understood. By small scales we mean the inertial range scales, i.e., those between the large scales where energy in injected into the fluid and the Kolmogorov scale where the kynetic energy is dissipated into heat. A. Chorin, in his book [5] and related works, suggests certain probabilistic descriptions of the vortex structures that are observed in a number of experiments. His theories throw light on the possible geometry of these structures and their statistics. One of the main purposes of a statistical description of small scale structures is to recover, or correct, statistical laws of turbulent flows, like the well-known Kolmogorov-Obukov law for the structure function and energy spectrum in the inertial range. The probabilistic models proposed by Chorin are very attractive and capture many aspects of the problem; some other aspects are less clearly understood, and our aim here is to discuss one of the remaining difficulties and show some partial positive results. Part of the intuitive idea contained in the present work has been in fact suggested by A. Chorin in private discussions. 
In general terms, one would like to define a space $\Omega$ of fluid configurations, with a $\sigma$ algebra $\mathcal{F}$ and a probability measure $\mu$, such that a number of requirements are fulfilled: the configurations have to be objects at least phenomenologically similar to the observed structures of 3D fluids; statistics like the energy spectrum should be similar to those measured in experiments, etc. In principle one should take a probability space $(\Omega, \mathcal{F}, \mu)$ rigorously arising from the Navier-Stokes equations (a set of velocity fields with an invariant mesure), but this complete program does not yield interesting quantitative results at present (see $[2,7-9,27]$ for theoretical results in this direction). If one believes, as it seems from several experiments, that the vorticity is concentrated in filament-like structures that dominate the small scale evolution, one can look for a phenomenological model based on vorticity curves (the elements of $\Omega$ ) endowed with a suitable probability measure. In analogy with statistical mechanics, a first attempt is to take a Gibbs measure with weigth

$$
\frac{1}{Z} \mathrm{e}^{-\beta H(\gamma)}
$$

where $H(\gamma)$ is the kynetic energy associated to the vorticity configuration $\gamma \in \Omega$. The choice of a Gibbs prescription for a vortex system is not obvious a priori, but it has some motivations and may lead to interesting results, see [5,18], and also references therein on statistical mechanics of two-dimensional point vortices.

One of the main technical problems in this approach is that the energy of a curve is infinite. This fact is easy and well known for smooth curves, see Section 2.1. One could hope that a fractal curve, like a Brownian trajectory, may have finite energy (the foldings of the curve reduce the energy, see [5], so the irregularity of the curve may play a positive role). However, the (natural) definition of energy given below shows that also Brownian trajectories have infinite energy. A theorem of Akao, see [1], also provides a certain evidence that any kind of vortex curve has infinite energy. On an informal ground (not reported here) we have also computed (part of ) the energy for a fractional Brownian trajectory for any Hurst parameter, and we have obtained divergences as well.

One of the basic models proposed in [5] is made of lattice vortex filaments. The lattice approximation provides, besides other advantages, the cut-off necessary to get a finite energy. The problem of the limit structures, as the lattice spacing goes to zero, remains open. Addressing this problem has been the main motivation of our work. The continuous-space structures should be more intrinsic objects, and provide more accurate small scale statistics.

As suggested by Chorin, and as it emerges from the previous negative results, the limit structures cannot reduce to single curves, but should include some sort of cross section. One can artificially define tubes around the curves which provide again a cutoff, but they are similar to lattice approximation from the view-point of the drawbacks. Moreover, a fractal cross section looks more convincing in comparison with certain numerical experiments, see for instance [3].

We introduce a very preliminary model based on vorticity fields concentrated over sets of the form

$$
C_{\mathcal{A}}=\left\{x+W_{t} ; x \in \mathcal{A}, t \in[0, T]\right\}
$$


where $\left(W_{t}\right)_{t \in[0, T]}$ is a Brownian motion in $\mathbf{R}^{3}$, and $\mathcal{A} \subset \mathbf{R}^{3}$ is a compact set supporting a probability measure $\rho$ such that

$$
\int_{\mathcal{A}} \int_{\mathcal{A}} \frac{1}{|x-y|} \rho(\mathrm{d} x) \rho(\mathrm{d} y)<\infty .
$$

As we shall see later, a certain interaction energy $H_{x y}$ behaves as $\frac{1}{|x-y|}$, so (2) will be the natural condition to have finite total energy. Condition (2) is well known in potential theory (see [13]). As we recall in the next section, every compact set $\mathcal{A}$ with positive capacity supports a probability measure $\rho$ satisfying (2). In particular, this is true for every compact set $\mathcal{A}$ with Hausdorff dimension strictly greater than one. The geometry of the sets $C_{\mathcal{A}}$ is certainly unrealistic to model regions of high vorticity; for instance, one expects that the cross section varies with the position along the filament. However, we believe that one can find generalization of the random sets $C_{\mathcal{A}}$, possibly based on the notion of random attractor (see [6]), with more realistic features.

We prove that the energy of vorticity configurations based on sets $C_{\mathcal{A}}$ is finite. Our analysis is based on the control of the mutual interaction between the (close but different) Brownian curves $x+W_{t}$ and $y+W_{t}$ with $x \neq y$. The interaction energy $H_{x y}$ is formally given by the double Stratonovich integral

$$
H_{x y}=\frac{\Gamma^{2}}{8 \pi} \int_{0}^{T} \int_{0}^{T} \frac{1}{\left|x+W_{t}-\left(y+W_{s}\right)\right|} \circ \mathrm{d} W_{s} \circ \mathrm{d} W_{t} .
$$

Our first result is a rigorous definition of $H_{x y}$. Then, roughly speaking, we prove that the interaction energy $H_{x y}$ diverges as

$$
H_{x y} \sim \frac{1}{|x-y|} .
$$

The self interaction $(x=y)$ would give an infinite contribution to the energy, but the total energy is the $\rho$-average of the mutual interactions, which is finite by assumption (2).

A remarkable fact is that the interaction energy contains, as one of the two dominating terms, the intersection local time of the 3-dimensional Brownian motion. This shows rigorously the expected importance of the self-intersections in the computation of the energy. When the Gibbs measure (1) will be considered, the penalization of self-intersections of polymer theories (Westwater [28], Bolthausen [4], etc.) will be automatically taken into account. In this connection it is important to understand whether other processes with less self-intersections may yield better results (see, for instance, Toth and Werner [26]).

To summarize, the three informations on Brownian vortex structures provided by this paper are a rigorous definition of $H_{x y}$ for $x \neq y$, the scaling of $H_{x y}$ as $|x-y| \rightarrow 0$ (and therefore the finiteness of the total energy under assumption (2)), and the relation with the intersection local time.

Although the energy of a single curve is infinite (we need the cross section $\mathcal{A}$ to have finite energy), there is still the possibility that a Gibbs ensemble $\mu_{\beta}$ for single 
curves is well defined by a renormalization procedure (namely when the distribution $\rho$ tends to a Dirac mass). This direction of research is further motivated by the similarity of the present problem with polymers and the success of renormalization for them, see $[28,4]$. More precisely, the decomposition proved in this paper suggests that the dominating divergencies are similar to those of polymers and only the double Itô integral of Definition 4 below seems to contain new terms. Nonetheless, the arguments of [28,4] cannot be easily extended since it would require the positivity of the interaction energy between different parts of a filament, a fact that is not true. So the renormalization of vortex filaments remains an open problem.

Once the space of configurations $\Omega$ and the energy of configurations is well defined, it is possible to address many questions concerning the Gibbs measures, the computation of important moments as the structure function, the existence of an Hamiltonian dynamic and its relations with Euler equation (see, for instance, [17] in two dimensions), the existence of Glauber type dynamics and their use for simulations, some form of invariance principle in connection with the theory of [5], and not last the attempt to go back to a single filament by renormalization. As we have remarked above, it is also very important to look for more realistic models, not based on a fixed set $\mathcal{A}$ and perhaps not on Brownian motion. All these problems contain nontrivial points and are open at present, but we hope to report on some of them in future works.

Let us finally remark that also the work of Gallavotti [10], Section II.11, has been a source of motivation for the present investigation, as well as the results of P.L. Lions and A. Majda [18] reported also in [16].

\section{Preliminary formal considerations}

\subsection{Formal expression of the energy}

The kynetic energy $H=\frac{1}{2} \int_{\mathbf{R}^{3}}|u(x)|^{2} \mathrm{~d} x$ of a velocity field $u(x)$ can be written, under suitable regularity and decay assumptions, in terms of the vorticity field $\xi(x)=\operatorname{curl} u(x)$ as (see [5])

$$
H=\frac{1}{8 \pi} \iint_{\mathbf{R}^{3}} \frac{\xi(x) \cdot \xi\left(x^{\prime}\right)}{\left|x-x^{\prime}\right|} \mathrm{d} x \mathrm{~d} x^{\prime} .
$$

In the ideal case of a vorticity field concentrated along a curve $\gamma(t), t \in[0, T]$, i.e. a field formally defined as (the parameter $\Gamma$ is the circuitation)

$$
\xi(x)=\Gamma \int_{0}^{T} \delta(x-\gamma(t)) \dot{\gamma}(t) \mathrm{d} t,
$$

the energy formally takes the form

$$
H=\frac{\Gamma^{2}}{8 \pi} \int_{0}^{T} \int_{0}^{T} \frac{\dot{\gamma}(t) \cdot \dot{\gamma}(s)}{|\gamma(t)-\gamma(s)|} \mathrm{d} t \mathrm{~d} s .
$$


For smooth curves this integral contains a non-integrable divergence along the diagonal.

Nonregular curves can be better, in principle. The term $|\gamma(t)-\gamma(s)|$ may be infinitesimal of order less than one (so $\frac{1}{|\gamma(t)-\gamma(s)|}$ is less divergent than in the smooth case), and very fast changes in direction may produce further cancellation in the term $\dot{\gamma}(t) \cdot \dot{\gamma}(s)$. Unfortunately this naïve hope is not confirmed by rigorous computations until now; the problems partially come from the very frequent self-intersections and for the major part it seems to come from the integrability requirements on $\frac{1}{|\gamma(t)-\gamma(s)|}$ imposed by the stochastic or generalized integrals appearing in $H$.

In order to construct vorticity fields with finite energy but still with an appealing fractal structure and suitable for a probabilistic treatment, we consider a distributional vorticity field formally expressed as

$$
\xi(x)=\Gamma \int_{\mathcal{A}}\left(\int_{0}^{T} \delta\left(x-y-W_{t}\right) \circ \mathrm{d} W_{t}\right) \rho(\mathrm{d} y)
$$

where $\mathcal{A}$ is a compact set in $\mathbf{R}^{3}, \rho$ is a probability measure supported by $\mathcal{A}$, and $\left(W_{t}\right)_{t \in[0, T]}$ is a Brownian motion in $\mathbf{R}^{3}$. For a rigorous definition see Section 2.2. The corresponding kynetic energy, in analogy with (3) and (5) (compare (4) and (6)) takes the form

$$
H=\frac{\Gamma^{2}}{8 \pi} \int_{\mathcal{A}} \int_{\mathcal{A}}\left(\int_{0}^{T} \int_{0}^{T} \frac{1}{\left|x+W_{t}-\left(y+W_{s}\right)\right|} \circ \mathrm{d} W_{s} \circ \mathrm{d} W_{t}\right) \rho(\mathrm{d} x) \rho(\mathrm{d} y) .
$$

If we introduce the interaction energy between the curves $\left(x+W_{t}\right)_{t \in[0, T]}$ and $(y+$ $\left.W_{t}\right)_{t \in[0, T]}$, with $x \neq y$, formally defined as

$$
H_{x y}=\frac{\Gamma^{2}}{8 \pi} \int_{0}^{T} \int_{0}^{T} \frac{1}{\left|x+W_{t}-\left(y+W_{s}\right)\right|} \circ \mathrm{d} W_{s} \circ \mathrm{d} W_{t},
$$

then we have

$$
H=\int_{\mathcal{A}} \int_{\mathcal{A}} H_{x y} \rho(\mathrm{d} x) \rho(\mathrm{d} y) .
$$

The purpose of this paper is to show that (7) has a meaningful definition, and (8) is also well defined under the assumption (2). Notice that for smooth curves $\gamma(t)$ in place of $W_{t}$, the interaction energy $H_{x y}$ can be infinite; however, this flaw of the smooth case has to be compared with the open problems mentioned in Remark 8.

Concerning condition (2), let us recall a few facts from potential theory, see [13]. A probability measure $\rho$ satisfying (2) is called a measure with finite energy (we restrict the attention to space dimension 3 and the Riesz kernel $\frac{1}{|x|}$, so we take $p=3$ and $\alpha=2$ in the notations of [13]). The capacity of a compact set $\mathcal{A}$ is the nonnegative number $W(\mathcal{A})^{-1}$, where $W(\mathcal{A})$ is the infimum of the double integral in (2), over all probability measures $\rho$ supported by $\mathcal{A}$, with the obvious convention $W(\mathcal{A})^{-1}=0$ when no such 
measures exist. Therefore, by definition, there exists a probability measure $\rho$ supported by $\mathcal{A}$ with finite energy if and only if the capacity of $\mathcal{A}$ is strictly positive. In such a case the infimum of the double integral in (2) is reached by a probability measure, proportional to the so-called equilibrium measure, which therefore provides a sort of canonical example of measure satisfying (2). Finally, by Theorem 3.13 of [13], every compact set with Hausdorff dimension $d>1$ has positive capacity. Therefore, it supports a probability measure $\rho$ satisfying (2).

\subsection{Motivations for the Stratonovich integration}

From the viewpoint of fluid dynamics the choice (6) for a vorticity field is artificial for a number of reasons, so it has to be considered only as a first step. A part from the major question of the physical realism, one problem with (6) is that it does not fulfill the necessary constraint $\operatorname{div} \xi(x)=0$ of incompressible fluid dynamics, the one we deal with here. To remedy this problem we should either consider closed curves (thus a Brownian bridge) or curves with parameter $t$ over all $\mathbf{R}$. Both ways complicate considerably the analysis, and probably they would not lead to very different quantitative results as far as interesting mean values are concerned, so for sake of simplicity we restrict the attention to the artificial case above. However, we keep in mind the constraint $\operatorname{div} \xi(x)=0$ in the fact that we take Stratonovich integrals, as we show below. The choice of Stratonovich integrals is more natural also from the viewpoint of the approximation by regular fields (a problem not treated here). In addition, if we replace Stratonovich integrals by Itô ones in (8) above we formally get $E[H]=0$ which is physically meaningless.

On the other side, the choice of Stratonovich integrals is the origin of certain technical difficulties, since we have to understand the correction terms in the Itô formulation. However, we shall also discover the interesting fact that one of the correction terms is the intersection local time.

Remark 1. - Neglecting the condition $\operatorname{div} \xi(x)=0$ we are not able to prove $H \geqslant 0$. We only have $E[H]>0$.

Let us see the relation between the Stratonovich integration and the condition $\operatorname{div} \xi(x)=0$. Let $\left(W_{t}\right)_{t \in \mathbf{R}}$ be a two-sided Brownian motion in $\mathbf{R}^{3}$ (defined for instance as a forward Brownian motion for $t \geqslant 0$ glued with a backward Brownian motion for $t \leqslant 0$, both equal to zero for $t=0$, adapted to the filtration $\mathcal{F}_{t}=\sigma\left\{W_{s}-W_{r} ; r \leqslant s \leqslant t\right\}$ ). Consider the distributional vorticity field

$$
\xi(x)=\int_{\mathcal{A}}\left(\int_{-\infty}^{+\infty} \delta\left(x-y-W_{t}\right) \circ \mathrm{d} W_{t}\right) \rho(\mathrm{d} y) .
$$

The rigorous definition is the following one: for all vector fields $\phi: \mathbf{R}^{3} \rightarrow \mathbf{R}^{3}$, of class $C^{\infty}$ with compact support (shortly $\phi \in \mathcal{D}\left(\mathbf{R}^{3}, \mathbf{R}^{3}\right)$ ), we set

$$
\xi(\phi)=\int_{\mathcal{A}}\left(\int_{-\infty}^{+\infty} \phi\left(y+W_{t}\right) \circ \mathrm{d} W_{t}\right) \rho(\mathrm{d} y) .
$$


Thanks to the transience of $W$ in $\mathbf{R}^{3}$ and the compact support of $\phi$, the integration $\int_{-\infty}^{+\infty}$ reduces to an integration over a random bounded interval; the integration is understood in the Stratonovich form, and the scalar product of the vector fields $\phi\left(x^{\prime}+W_{t}\right)$ and $\mathrm{d} W_{t}$ is taken; the Stratonovich integral is well defined since $\phi\left(x^{\prime}+W_{t}\right)$ is a semimartingale; all the other operations on the right-hand-side of (9) are well defined, so $\xi(\phi)$ is a real valued random variable. In this sense, as an operator from $\mathcal{D}\left(\mathbf{R}^{3}, \mathbf{R}^{3}\right)$ to the set of random variables, $\xi(\phi)$ is a generalized random field.

For all smooth scalar fields $q$ with compact support, we have

$$
\begin{aligned}
\xi(\nabla q) & =\int_{\mathcal{A}}\left(\int_{-\infty}^{+\infty} \nabla q\left(y+W_{t}\right) \circ \mathrm{d} W_{t}\right) \rho(\mathrm{d} y) \\
& =\int_{\mathcal{A}}\left(\lim _{T \rightarrow \infty}\left[q\left(y+W_{t}\right)\right]_{t=-T}^{t=T}\right) \rho(\mathrm{d} y)=0 .
\end{aligned}
$$

This means $\operatorname{div} \xi(x)=0$ in the sense of distributions. The same property fails if we take an Itô integral. The definitions and results of this section are not used below, so we have taken the freedom to express them in a slightly informal way.

\subsection{Difficulties and strategy to define the energy}

A rigorous definition of the energies (7) and (8) has to overcome the following difficulties:

- The double stochastic integral in (7) contains anticipative integrands.

- The Itô formulation of the Stratonovich integrals introduces correction terms with singularities of higher order than $\frac{1}{\left|x+W_{t}-\left(y+W_{s}\right)\right|}$.

To deal with the first difficulty we do not need to use anticipative calculus. If we define rigorously "half" of the interaction energy, namely the quantity

$$
H_{x y}^{\left(\frac{1}{2}\right)}=\frac{\Gamma^{2}}{8 \pi} \int_{0}^{T}\left(\int_{0}^{t} \frac{1}{\left|x+W_{t}-\left(y+W_{s}\right)\right|} \circ \mathrm{d} W_{s}\right) \circ \mathrm{d} W_{t},
$$

then we set

$$
H_{x y}=H_{x y}^{\left(\frac{1}{2}\right)}+H_{y x}^{\left(\frac{1}{2}\right)} .
$$

To accept this decomposition (we cannot prove it, since we are still looking for a meaningful definition) notice that for smooth approximations $W_{t}^{n}$ of $W_{t}$, and a smooth approximation $\frac{1}{|.|_{\varepsilon}}$ of $\frac{1}{|.|}$, we have

$$
\begin{aligned}
\int_{0}^{T} & \left(\int_{t}^{T} \frac{1}{\left|x+W_{t}^{n}-\left(y+W_{s}^{n}\right)\right|_{\varepsilon}} \mathrm{d} W_{s}^{n}\right) \mathrm{d} W_{t}^{n} \\
& =\int_{0}^{T}\left(\int_{0}^{s} \frac{1}{\left|x+W_{t}^{n}-\left(y+W_{s}^{n}\right)\right|_{\varepsilon}} \mathrm{d} W_{t}^{n}\right) \mathrm{d} W_{s}^{n}
\end{aligned}
$$




$$
\begin{aligned}
& =\int_{0}^{T}\left(\int_{0}^{t} \frac{1}{\left|x+W_{s}^{n}-\left(y+W_{t}^{n}\right)\right|_{\varepsilon}} \mathrm{d} W_{s}^{n}\right) \mathrm{d} W_{t}^{n} \\
& =\int_{0}^{T}\left(\int_{0}^{t} \frac{1}{\left|y+W_{t}^{n}-\left(x+W_{s}^{n}\right)\right|_{\varepsilon}} \mathrm{d} W_{s}^{n}\right) \mathrm{d} W_{t}^{n} .
\end{aligned}
$$

As a second step, the double stochastic integral in (10) can be approached either with the theory of multiple stochastic integrals (at least after it has been rewritten in Itô form), see, for instance, [22], or (as we shall do below), by using backward integration in the internal integral. We interpet

$$
\int_{0}^{t} \frac{1}{\left|x+W_{t}-\left(y+W_{s}\right)\right|} \circ \mathrm{d} W_{s}
$$

in (10) as the backward Stratonovich integral (see [12]; the forward/backward calculus of Russo and Valois, [23,24], is also suitable for the following purposes)

$$
\int_{0}^{t} \frac{1}{\left|x+W_{t}-\left(y+W_{s}\right)\right|} \circ \hat{\mathrm{d}} W_{s} .
$$

To this end we recall that one has to introduce the $\sigma$-algebras

$$
\mathcal{F}_{s}^{t}=\sigma\left\{W_{\tau}-W_{r} ; s \leqslant r \leqslant \tau \leqslant t\right\}
$$

Given $t>0$, the family $\left(\mathcal{F}_{s}^{t}\right)_{s \in[0, t]}$ is a backward filtration $\left(\mathcal{F}_{s_{1}}^{t} \supset \mathcal{F}_{s_{2}}^{t}\right.$ if $\left.s_{1} \leqslant s_{2}\right)$, the process $\left(\tilde{W}_{s}^{t}\right)_{s \in[0, t]}$ defined as $\tilde{W}_{s}^{t}=W_{t}-W_{s}$ is a backward Brownian motion adapted to $\left(\mathcal{F}_{s}^{t}\right)_{s \in[0, t]}$, and one can integrate in the Itô sense processes $\left(X_{s}\right)_{s \in[0, t]}$ backward adapted to $\left(\mathcal{F}_{s}^{t}\right)_{s \in[0, t]}$, with integrability conditions of the same kind as for the forward integration theory. We refer to [12] for details on backward stochastic calculus. For instance, if $\left(X_{s}\right)_{s \in[0, t]}$ is a continuous process, $X_{s}$ measurable with respect to $\mathcal{F}_{s}^{t}$ for all $s \in[0, t]$, then the backward Itô integral $\int_{0}^{t} X_{s} \hat{\mathrm{d}} W_{s}$ is the limit in probability of the Riemann sums

$$
\int_{0}^{t} X_{s} \hat{\mathrm{d}} W_{s}=P-\lim _{n \rightarrow \infty} \sum_{\substack{s_{i} \in \pi_{n} \\ s_{i}<t}} X_{s_{i+1} \wedge t}\left(W_{s_{i+1} \wedge t}-W_{s_{i}}\right)
$$

where $\left\{\pi_{n}\right\}$ is a sequence of partitions with lengths going to zero. We have recalled these facts also to point out the convention of taking the increments $\left(W_{s_{i+1} \wedge t}-W_{s_{i}}\right)$ instead of $\left(W_{s_{i}}-W_{s_{i+1} \wedge t}\right)$, a difference in sign that has to be clarified in view of the computations of the next section. Similarly the backward Stratonovich integral $\int_{0}^{t} X_{S} \circ \hat{\mathrm{d}} W_{s}$ is well defined for instance when $\left(X_{s}\right)_{s \in[0, t]}$ is a continuous backward semimartingale with respect to 
$\left(\mathcal{F}_{s}^{t}\right)_{s \in[0, t]} ;$ in such a case

$$
\int_{0}^{t} X_{s} \circ \hat{\mathrm{d}} W_{s}=P-\lim _{n \rightarrow \infty} \sum_{\substack{s_{i} \in n_{n} \\ s_{i}<t}} \frac{X_{s_{i+1} \wedge t}+X_{s_{i}}}{2}\left(W_{s_{i+1} \wedge t}-W_{s_{i}}\right) .
$$

With these notations in mind, we shall give a rigorous definition of

$$
H_{x y}^{\left(\frac{1}{2}\right)}=\frac{\Gamma^{2}}{8 \pi} \int_{0}^{T}\left(\int_{0}^{t} \frac{1}{\left|x+W_{t}-\left(y+W_{s}\right)\right|} \circ \hat{\mathrm{d}} W_{s}\right) \circ \mathrm{d} W_{t} .
$$

This solves the first difficulty mentioned above (anticipative integrands). The second one (high singularities arising from the correction terms of Stratonovich integrals) is dealt with by means of the lemma of the next section. It is a careful way to rewrite the correction terms in order to identify the leading singularities. More naïve expressions produce higher or more obscure singularities which in fact should cancel each other, but it is not trivial to detect the cancellations.

\section{A preliminary identity}

Consider a smooth function $\sigma(x)$ from $\mathbf{R}^{3}$ to $\mathbf{R}$, with compact support (a good decay at infinity like $\sigma(u) \sim \frac{1}{|u|}$ and $|\nabla \sigma(u)| \sim \frac{1}{|u|^{2}}$ is sufficient, as well as the regularity $\left.\sigma \in C^{2}\left(\mathbf{R}^{3} ; \mathbf{R}\right)\right)$. Consider the double stochastic integral

$$
I=\int_{a}^{b}\left(\int_{a}^{t} \sigma\left(u+W_{t}-W_{s}\right) \circ \hat{\mathrm{d}} W_{s}\right) \circ \mathrm{d} W_{t}
$$

with the notations introduced before. Here we take a generic time interval $[a, b]$ (possibly with negative $a$, if we consider a two-sided Brownian motion), and $u$ is a given point in $\mathbf{R}^{3}$. This may be useful for future investigations on filaments with unbounded parameter range $t \in \mathbf{R}$.

The integral $I$ is well defined. Indeed, for every $t \in[a, b]$, the internal Stratonovich (backward) integral is well defined since $\sigma\left(u+W_{t}-W_{s}\right)$ is a backward continuous semimartingale with respect to $\left(\mathcal{F}_{s}^{t}\right)_{s \in[a, t]}$. The internal integral is forward progressively measurable with respect to $\left(\mathcal{F}_{a}^{t}\right)_{t \in[a, b]}$, from (12). Moreover, one has

$$
\sup _{t \in[a, b]} E\left[\left(\int_{a}^{t} \sigma\left(u+W_{t}-W_{s}\right) \circ \hat{\mathrm{d}} W_{s}\right)^{2}\right]<\infty
$$

since $\sigma$ is smooth with compact support (a decay at infinity like $\sigma(u) \sim \frac{1}{|u|}$ and $|\nabla \sigma(u)| \sim \frac{1}{|u|^{2}}$ is sufficient, see Section 4). However this property would allow us to define the external integral only in the Itô sense. The external Stratonovich integral is 
well defined because

$$
\left(\int_{a}^{t} \sigma\left(u+W_{t}-W_{s}\right) \circ \hat{\mathrm{d}} W_{s}\right)_{t \in[a, b]}
$$

is a (forward) continuous semimartingale with respect to $\left(\mathcal{F}_{a}^{t}\right)_{t \in[a, b]}$. The proof of this fact is embodied in step 1 of the following lemma (see in particular (15) and (17)).

The next lemma shows that the double Stratonovich integral is equal to a double Itô integral plus correction terms, carefully choosen to identify the main divergences in the application to (10). The term with $\triangle \sigma$ is remarkable for later considerations.

LEMMA 2. - We have

$$
\begin{aligned}
I= & \int_{a}^{b}\left(\int_{a}^{t} \sigma\left(u+W_{t}-W_{s}\right) \hat{\mathrm{d}} W_{s}\right) \mathrm{d} W_{t} \\
& -\frac{1}{4} \int_{a}^{b}\left(\int_{a}^{t}(\Delta \sigma)\left(u+W_{t}-W_{s}\right) \mathrm{d} s\right) \mathrm{d} t \\
& +\frac{1}{2} \int_{a}^{b} \sigma(u) \mathrm{d} t \\
& +\frac{1}{2} \int_{a}^{b}\left(\sigma\left(u+W_{t}-W_{a}\right)+\sigma\left(u+W_{b}-W_{t}\right)\right) \mathrm{d} t .
\end{aligned}
$$

Proof. - Step 1. The forward Stratonovich integral

$$
J_{t}(z)=\int_{a}^{t} \sigma\left(z-W_{s}\right) \circ \mathrm{d} W_{s}
$$

is well defined, since $\sigma\left(z-W_{s}\right)$ is a continuous semimartingale, and is the limit in probability (uniformly in $t$ over compact sets) of

$$
\sum_{\substack{s_{i} \in \pi_{n} \\ s_{i}<t}} \frac{\sigma\left(z-W_{s_{i+1} \wedge t}\right)+\sigma\left(z-W_{s_{i}}\right)}{2}\left(W_{s_{i+1} \wedge t}-W_{s_{i}}\right)
$$

where $\left\{\pi_{n}\right\}$ is a sequence of partitions with lengths going to zero. Therefore it is equal to the backward Stratonovich integral:

$$
J_{t}(z)=\int_{a}^{t} \sigma\left(z-W_{s}\right) \circ \hat{\mathrm{d}} W_{s} .
$$


By the regularity of $\sigma$ and the backward adaptedness (we use the representation (16)) we have

$$
J_{t}\left(u+W_{t}\right)=\int_{a}^{t} \sigma\left(u+W_{t}-W_{s}\right) \circ \hat{\mathrm{d}} W_{s} .
$$

Here we have used a substitution argument similar for instance to Corollary 7.9 of [12] for the Itô part, and a classical argument for the Riemann integral that gives us the Stratonovich correction.

Since (by (15))

$$
\mathrm{d} J_{t}(z)=\sigma\left(z-W_{t}\right) \circ \mathrm{d} W_{t},
$$

by Itô-Wentzell formula, $J_{t}\left(u+W_{t}\right)$ is also given by

$$
J_{t}\left(u+W_{t}\right)=\int_{a}^{t} \sigma(u) \circ \mathrm{d} W_{s}+\int_{a}^{t}(D J)_{s}\left(u+W_{s}\right) \circ \mathrm{d} W_{s}
$$

(in the latter stochastic integral we have a product matrix-vector). Therefore

$$
\begin{aligned}
I= & \int_{a}^{b}\left(\int_{a}^{t} \sigma\left(u+W_{t}-W_{s}\right) \circ \hat{\mathrm{d}} W_{s}\right) \mathrm{d} W_{t}+\frac{1}{2}[J(u+W), W]_{a}^{b} \\
= & \int_{a}^{b}\left(\int_{a}^{t} \sigma\left(u+W_{t}-W_{s}\right) \circ \hat{\mathrm{d}} W_{s}\right) \mathrm{d} W_{t} \\
& +\frac{3}{2} \int_{a}^{b} \sigma(u) \mathrm{d} t+\frac{1}{2} \int_{a}^{b}\left(\operatorname{div}_{z} J\right)_{t}\left(u+W_{t}\right) \mathrm{d} t
\end{aligned}
$$

because

$$
\begin{aligned}
{[J(u+W), W]_{a}^{b} } & =\sum_{i}\left[J^{i}(u+W), W^{i}\right]_{a}^{b} \\
& =\sum_{i} \int_{a}^{b} \sigma(u) \mathrm{d} t+\sum_{i} \sum_{j} \int_{a}^{b} \frac{\partial J_{t}^{i}}{\partial z_{j}}\left(u+W_{t}\right) \mathrm{d}\left[W^{j}, W^{i}\right]_{t}
\end{aligned}
$$

from (18).

Step 2. From (15) (since $\sigma \in C^{2}$, we can differentiate in $z$ under the integral, see [12]) we have

$$
\begin{aligned}
\left(\operatorname{div}_{z} J\right)_{t}(z) & =\sum_{i} \int_{a}^{t}\left(\frac{\partial \sigma}{\partial z_{i}}\right)\left(z-W_{s}\right) \circ \mathrm{d} W_{s}^{i}=\int_{a}^{t}(\nabla \sigma)\left(z-W_{s}\right) \circ \mathrm{d} W_{s} \\
& =-\sigma\left(z-W_{t}\right)+\sigma\left(z-W_{a}\right),
\end{aligned}
$$

so

$$
\left(\operatorname{div}_{z} J\right)_{t}\left(u+W_{t}\right)=-\sigma(u)+\sigma\left(u+W_{t}-W_{a}\right) .
$$


Hence

$$
\int_{a}^{b}\left(\operatorname{div}_{z} J\right)_{t}\left(u+W_{t}\right) \mathrm{d} t=\int_{a}^{b}\left[-\sigma(u)+\sigma\left(u+W_{t}-W_{a}\right)\right] \mathrm{d} t,
$$

which implies

$$
\begin{aligned}
I= & \int_{a}^{b}\left(\int_{a}^{t} \sigma\left(u+W_{t}-W_{s}\right) \circ \hat{\mathrm{d}} W_{s}\right) \mathrm{d} W_{t} \\
& +\int_{a}^{b} \sigma(u) \mathrm{d} t+\frac{1}{2} \int_{a}^{b} \sigma\left(u+W_{t}-W_{a}\right) \mathrm{d} t .
\end{aligned}
$$

Step 3. Let us now transform the internal Stratonovich integral. From the rules of backward calculus ([12]) we have

$$
\begin{aligned}
\int_{a}^{t} \sigma\left(u+W_{t}-W_{s}\right) \circ \hat{\mathrm{d}} W_{s} \\
\quad=\int_{a}^{t} \sigma\left(u+W_{t}-W_{s}\right) \hat{\mathrm{d}} W_{s}-\frac{1}{2}\left[\sigma\left(u+W_{t}-W_{.}\right), W_{.}\right]_{a}^{t} \\
\quad=\int_{a}^{t} \sigma\left(u+W_{t}-W_{s}\right) \hat{\mathrm{d}} W_{s}+\frac{1}{2} \int_{a}^{t}(\nabla \sigma)\left(u+W_{t}-W_{s}\right) \mathrm{d} s .
\end{aligned}
$$

Since the positive sign of the second term is very important for the sequel, we simply recall that the rules of backward calculus used above is based on the identity

$$
\begin{aligned}
\sum_{\substack{s_{i} \in \pi_{n} \\
s_{i}<t}} & \frac{\sigma\left(u+W_{t}-W_{s_{i+1} \wedge t}\right)+\sigma\left(u+W_{t}-W_{s_{i}}\right)}{2}\left(W_{s_{i+1} \wedge t}-W_{s_{i}}\right) \\
= & \sum_{\substack{s_{i} \in \pi_{n} \\
s_{i}<t}} \sigma\left(u+W_{t}-W_{s_{i+1} \wedge t}\right)\left(W_{s_{i+1} \wedge t}-W_{s_{i}}\right) \\
& -\sum_{\substack{s_{i} \in \pi_{n} \\
s_{i}<t}} \frac{\sigma\left(u+W_{t}-W_{s_{i+1} \wedge t}\right)-\sigma\left(u+W_{t}-W_{s_{i}}\right)}{2}\left(W_{s_{i+1} \wedge t}-W_{s_{i}}\right) .
\end{aligned}
$$

The second term of (19) inserted into the external integral gives us (by the Stochastic Fubini theorem, see [29] for similar computations)

$$
\int_{a}^{b}\left(\frac{1}{2} \int_{a}^{t}(\nabla \sigma)\left(u+W_{t}-W_{s}\right) \mathrm{d} s\right) \mathrm{d} W_{t}=\frac{1}{2} \int_{a}^{b}\left(\int_{s}^{b}(\nabla \sigma)\left(u+W_{t}-W_{s}\right) \mathrm{d} W_{t}\right) \mathrm{d} s .
$$

But 


$$
\begin{aligned}
\sigma\left(u+W_{b}-W_{s}\right)= & \sigma(u)+\int_{s}^{b}(\nabla \sigma)\left(u+W_{t}-W_{s}\right) \cdot \mathrm{d} W_{t} \\
& +\frac{1}{2} \int_{s}^{b}(\Delta \sigma)\left(u+W_{t}-W_{s}\right) \mathrm{d} t .
\end{aligned}
$$

Hence

$$
\begin{aligned}
\int_{a}^{b}\left(\frac{1}{2} \int_{a}^{t}(\nabla \sigma)\left(u+W_{t}-W_{s}\right) \mathrm{d} s\right) \mathrm{d} W_{t} \\
=\frac{1}{2} \int_{a}^{b}\left(\sigma\left(u+W_{b}-W_{s}\right)-\sigma(u)-\frac{1}{2} \int_{s}^{b}(\Delta \sigma)\left(u+W_{t}-W_{s}\right) \mathrm{d} t\right) \mathrm{d} s \\
=-\frac{1}{4} \int_{a}^{b}\left(\int_{a}^{t}(\Delta \sigma)\left(u+W_{t}-W_{s}\right) \mathrm{d} s\right) \mathrm{d} t \\
\quad-\frac{1}{2} \int_{a}^{b} \sigma(u) \mathrm{d} t+\frac{1}{2} \int_{a}^{b} \sigma\left(u+W_{b}-W_{s}\right) \mathrm{d} s .
\end{aligned}
$$

In conclusion, we have

$$
\begin{aligned}
I= & \int_{a}^{b}\left(\int_{a}^{t} \sigma\left(u+W_{t}-W_{s}\right) \hat{\mathrm{d}} W_{s}\right) \mathrm{d} W_{t}+\int_{a}^{b}\left(\frac{1}{2} \int_{a}^{t}(\nabla \sigma)\left(u+W_{t}-W_{s}\right) \mathrm{d} s\right) \mathrm{d} W_{t} \\
& +\int_{a}^{b} \sigma(u) \mathrm{d} t+\frac{1}{2} \int_{a}^{b} \sigma\left(u+W_{t}-W_{a}\right) \mathrm{d} t \\
= & \int_{a}^{b}\left(\int_{a}^{t} \sigma\left(u+W_{t}-W_{s}\right) \hat{\mathrm{d}} W_{s}\right) \mathrm{d} W_{t}-\frac{1}{4} \int_{a}^{b}\left(\int_{a}^{t}(\Delta \sigma)\left(u+W_{t}-W_{s}\right) \mathrm{d} s\right) \mathrm{d} t \\
& +\frac{1}{2} \int_{a}^{b} \sigma(u) \mathrm{d} t+\frac{1}{2} \int_{a}^{b}\left(\sigma\left(u+W_{t}-W_{a}\right)+\sigma\left(u+W_{b}-W_{t}\right)\right) \mathrm{d} t .
\end{aligned}
$$

The proof is complete.

Remark 3. - Yor's version [29] of Rosen-Tanaka formula includes computations of the form (20), (21) and (22), in the reversed order: the aim of [29] is is to express the difficult integral $\int_{a}^{b} \int_{a}^{t}(\Delta \sigma)\left(u+W_{t}-W_{s}\right) \mathrm{d} s \mathrm{~d} t$ in terms of easier integrals. Our motivation here to apply a reversed procedure with respect to [29] is to put in evidence the dominating singularities and display the (conceptually important) presence of the intersection local time, deeply investigated in the literature. 


\section{Definitions}

Recall that (in the sense of distributions)

$$
\frac{1}{4 \pi} \triangle \frac{1}{|x|}=-\delta(x)
$$

The identity of the previous section, along with the first steps indicated in previous sections, motivate the following definition (we replace $\sigma(x)$ by $\frac{1}{|x|}$ ).

Definiton 4. - We call interaction energy, over the parameter interval [0, T], between the vortex filaments $\left(x+W_{t}\right)_{t \in[0, T]}$ and $\left(y+W_{t}\right)_{t \in[0, T]}$ with $x \neq y$, where $\left(W_{t}\right)_{t \in[0, T]}$ is a Brownian motion in $\mathbf{R}^{3}$, the expression $H_{x y}$ defined as

$$
H_{x y}=H_{x y}^{\left(\frac{1}{2}\right)}+H_{y x}^{\left(\frac{1}{2}\right)}
$$

where

$$
\begin{gathered}
\frac{8 \pi}{\Gamma^{2}} H_{x y}^{\left(\frac{1}{2}\right)}=I_{1}(x-y)+I_{2}(x-y)+I_{3}(x-y)+I_{4}(x-y), \\
I_{1}(x-y)=\int_{0}^{T}\left(\int_{0}^{t} \frac{1}{\left|x+W_{t}-\left(y+W_{s}\right)\right|} \hat{\mathrm{d}} W_{s}\right) \mathrm{d} W_{t}, \\
I_{2}(x-y)=\pi \alpha(x-y ; T), \\
I_{3}(x-y)=\frac{1}{2} \int_{0}^{T} \frac{1}{|x-y|} \mathrm{d} t, \\
I_{4}(x-y)=\frac{1}{2} \int_{0}^{T}\left(\frac{1}{\left|x+W_{t}-y\right|}+\frac{1}{\left|x+W_{T}-\left(y+W_{t}\right)\right|}\right) \mathrm{d} t,
\end{gathered}
$$

and where $\alpha(u ; T), u \in \mathbf{R}^{3}$, is the intersection local time of the Brownian motion $\left(W_{t}\right)$, formally given by

$$
\alpha(u ; T)=\int_{0}^{T}\left(\int_{0}^{t} \delta\left(u+W_{t}-W_{s}\right) \mathrm{d} s\right) \mathrm{d} t .
$$

The intersection local time of the Brownian motion has been rigorously defined by Rosen [20,21], and further studied by many authors, including Le Gall [14,15], Yor [29, 30], Imkeller et al. [11], works that contain relevant results for the sequel. The first theorem below states that the previous definition of $H_{x y}$ is meaningful. The second theorem deals with the divergence of $H_{x y}$ as $|x-y| \rightarrow 0$. Let us anticipate the final definition of energy. 
Definiton 5. - We call kynetic energy of the vortex structure $\left(C_{\mathcal{A}}, \rho\right)$, where $C_{\mathcal{A}}$ is the random set $\left\{x+W_{t} ; x \in \mathcal{A}, t \in[0, T]\right\}$ with $\left(W_{t}\right)_{t \in[0, T]}$ a Brownian motion in $\mathbf{R}^{3}$ and $\mathcal{A} \subset \mathbf{R}^{3}$ a compact set, and where $\rho$ is a probability measure supported by $\mathcal{A}$ such that (2) holds true, the expression

$$
H=\int_{\mathcal{A}} \int_{\mathcal{A}} H_{x y} \rho(\mathrm{d} x) \rho(\mathrm{d} y)
$$

The third theorem below states that this definition is meaningful, and $H$ is finite.

THEOREM 6. - For every $x \neq y, H_{x y}$ is a well defined real valued random variable, with finite moments of every order. The family $\left\{H_{x y} ; x, y \in \mathbf{R}^{3}\right\}$ is a measurable random field; more precisely, $\left\{I_{1}(x-y) ; x, y \in \mathbf{R}^{3}\right\}$ is a measurable random field, $\left\{\alpha(x-y ; T) ; x, y \in \mathbf{R}^{3}, x \neq y\right\}$ is a continuous random field, $\left\{I_{4}(x-y) ; x, y \in \mathbf{R}^{3}\right\}$ is a continuous random field (see steps 2 and 3 for further regularity of these fields).

Proof. - Step 1. In this step we discuss the properties of $I_{1}(x-y)$. First, let $x \neq y$ be given. The internal backward integral

$$
\int_{0}^{t} \frac{1}{\left|x+W_{t}-\left(y+W_{s}\right)\right|} \hat{\mathrm{d}} W_{s}
$$

is well defined, for every given $t$, since the process

$$
\left(\frac{1}{\left|x+W_{t}-\left(y+W_{s}\right)\right|}\right)_{s \in[0, T]}
$$

is $P$-a.s. continuous (the point $x-y$ is polar) and adapted to the filtration $\left(\mathcal{F}_{s}^{t}\right)_{s \in[0, t]}$. In addition, we shall see below that

$$
\sup _{t \in[0, T]} E \int_{0}^{t} \frac{1}{\left|x+W_{t}-\left(y+W_{s}\right)\right|^{2}} \mathrm{~d} s<\infty
$$

so that the internal integral is of class $L_{P}^{2}$. Since the internal integral is the $L_{P}^{2}$-limit of Riemann sums, see (11), it is easy to check that it is jointly measurable in $(t, \omega)$, and also progressively measurable with respect to the filtration $\left(\mathcal{F}_{0}^{t}\right)_{t \in[0, T]}$. Therefore, from (23), it follows that also the external stochastic integral is well defined and is of class $L_{P}^{2}$. Concerning the joint measurability also in the space variable $x-y$, it follows from a simple limit argument when $\frac{1}{\left|x+W_{t}-\left(y+W_{s}\right)\right|}$ is approximated by $\frac{1}{\left|x+W_{t}-\left(y+W_{s}\right)\right|_{\varepsilon}}$ where $\frac{1}{|\cdot|_{\varepsilon}}$ denotes a smooth compact support approximation of $\frac{1}{|.|}$.

Let us prove that $I_{1}(x-y)$ is of class $L_{P}^{2 n}$ for all positive integers $n$, when $x \neq y$ are given. Using the Burkholder-Davis-Gundy inequality, we have (we use obvious notations for the constants, without further comments) 


$$
\begin{aligned}
& E\left[\left|\int_{0}^{T}\left(\int_{0}^{t} \frac{1}{\left|x+W_{t}-\left(y+W_{s}\right)\right|} \hat{\mathrm{d}} W_{s}\right) \mathrm{d} W_{t}\right|^{2 n}\right] \\
& \leqslant C_{n} E\left[\left|\int_{0}^{T}\left(\int_{0}^{t} \frac{1}{\left|x+W_{t}-\left(y+W_{s}\right)\right|} \hat{\mathrm{d}} W_{s}\right)^{2} \mathrm{~d} t\right|^{n}\right] \\
& \leqslant C_{n, T} \int_{0}^{T} E\left[\left(\int_{0}^{t} \frac{1}{\left|x+W_{t}-\left(y+W_{s}\right)\right|} \hat{\mathrm{d}} W_{s}\right)^{2 n}\right] \mathrm{d} t \\
& \leqslant C_{n} C_{n, T} \int_{0}^{T} E\left[\left(\int_{0}^{t} \frac{1}{\left|x+W_{t}-\left(y+W_{s}\right)\right|^{2}} \mathrm{~d} s\right)^{n}\right] \mathrm{d} t .
\end{aligned}
$$

Notice that the constants $C_{n}$ and $C_{n, T}$ diverge, as $n \rightarrow \infty$, as $L^{n} n^{2 n}$, for some $L>0$. Now the random variable

$$
\int_{0}^{t} \frac{1}{\left|x+W_{t}-\left(y+W_{s}\right)\right|^{2}} d s,
$$

with $x \neq y$, is known to have finite moments to all orders, see Yor [29], p. 345, due to the identity (obtained from the Itô formula for $d_{s} \log \left|x+W_{t}-\left(y+W_{s}\right)\right|$ )

$$
\begin{aligned}
\log \left|x+W_{t}-y\right|-\log |x-y|= & \int_{0}^{t} \frac{1}{\left|x+W_{t}-\left(y+W_{s}\right)\right|} \mathrm{d} \beta_{s} \\
& -\frac{1}{2} \int_{0}^{t} \frac{1}{\left|x+W_{t}-\left(y+W_{s}\right)\right|^{2}} \mathrm{~d} s
\end{aligned}
$$

where $\left(\beta_{t}\right)$ is an auxiliary one-dimensional Brownian motion. We give another proof, along the lines of Khasminskii lemma, [21, p. 146], [25, p. 8], since this is another recurrent theme in the sequel. Denote by $\left(Z_{t}\right)_{t \geqslant 0}$ the canonical process over the Wiener space $C\left([0, \infty) ; \mathbf{R}^{3}\right)$, and denote by $E_{x}$ the expectation with respect to the Wiener measure $P_{x}$ of the Brownian motion starting from $x \in \mathbf{R}^{3}$. Let us set $\log _{+} r=0 \vee \log r$. Preliminary, for every $\gamma \in(0,3), k \geqslant 0$, and $u, x \in \mathbf{R}^{3}, u+x \neq 0$, we have

$$
\begin{aligned}
E_{x} \int_{0}^{T} \frac{\log _{+}^{k} \frac{1}{\left|u+Z_{t}\right|}}{\left|u+Z_{t}\right|^{\gamma}} \mathrm{d} t & =\int_{0}^{T} \int_{\mathbf{R}^{3}} \frac{\log _{+}^{k} \frac{1}{|u+x+v|}}{|u+v|^{\gamma}} \frac{1}{\sqrt{(2 \pi t)^{3}}} \mathrm{e}^{-\frac{|v|^{2}}{2 t}} \mathrm{~d} v \mathrm{~d} t \\
& =\int_{\mathbf{R}^{3}} \frac{\log _{+}^{k} \frac{1}{|u+x+v|}}{|u+x+v|^{\gamma}} \int_{0}^{T} \frac{1}{\sqrt{(2 \pi t)^{3}}} \mathrm{e}^{-\frac{|v|^{2}}{2 t}} \mathrm{~d} t \mathrm{~d} v \\
& =\int_{\mathbf{R}^{3}} \frac{\log _{+}^{k} \frac{1}{|u+x+v|}}{|u+v|^{\gamma}|v|} \int_{\frac{|v|^{2}}{2 T}}^{\infty} \frac{\mathrm{e}^{-s}}{2 \sqrt{s} \sqrt{\pi^{3}}} \mathrm{~d} s \mathrm{~d} v
\end{aligned}
$$




$$
\begin{aligned}
& \leqslant c_{T} \int_{\mathbf{R}^{3}} \frac{\log _{+}^{k} \frac{1}{|u+x+v|}}{|u+x+v|^{\gamma}|v|} \mathrm{e}^{-\frac{|v|^{2}}{4 T}} \mathrm{~d} v \\
& \left.\quad \text { (since } \int_{\frac{|v|^{2}}{2 T}}^{\infty} \frac{\mathrm{e}^{-s}}{\sqrt{s}} \mathrm{~d} s \leqslant c+c \int_{\frac{|v|^{2}}{2 T}}^{\infty} \mathrm{e}^{-\frac{s}{2}} \mathrm{~d} s\right) \\
& \leqslant c_{T}^{\prime}\left(1+|u+x|^{2-\gamma} \log _{+}^{k+1} \frac{1}{|u+x|}\right) .
\end{aligned}
$$

The last inequality requires a few computations, but it can be shortly explained as follows. For $|u+x| \geqslant 1$ it is very easy. For $|u+x|<1$, one can reduce the problem to estimate the integral over $|v| \leqslant 2$, and further to estimate the integral

$$
\int_{|v| \leqslant 2} \frac{\log _{+}^{k} \frac{1}{\left|t e_{1}+v\right|}}{\left|t e_{1}+v\right|^{\gamma}|v|} \mathrm{d} v
$$

where, without loss of generality, $e_{1}=(1,0,0), t=|u+x| \in(0,1)$. By the change of variable $v=t z$ this is bounded by

$$
t^{2-\gamma} \int_{|z| \leqslant \frac{2}{t}} \frac{\left(\log \frac{1}{|t|}+\log _{+} \frac{1}{\left|e_{1}+z\right|}\right)^{k}}{\left|e_{1}+z\right|^{\gamma}|z|} \mathrm{d} z .
$$

As $t \rightarrow 0$ one can replace asymptotically $e_{1}+z$ by $z$, and then use spherical coordinates to obtain the desired bound.

Now we proceed as in the proof of Khasminskii lemma to estimate the moments of the integral of interest to us:

$$
\begin{aligned}
E & {\left[\left(\int_{0}^{t} \frac{1}{\left|x+W_{t}-\left(y+W_{s}\right)\right|^{2}} \mathrm{~d} s\right)^{n}\right] } \\
& \stackrel{u=x-y}{=} E_{0}\left[\left(\int_{0}^{t} \frac{1}{\left|u+Z_{s}\right|^{2}} \mathrm{~d} s\right)^{n}\right] \\
= & n ! \int_{0 \leqslant s_{1} \leqslant \cdots \leqslant s_{n} \leqslant t} E_{0}\left[\frac{1}{\left|u+Z_{s_{1}}\right|^{2}} \cdots \frac{1}{\left|u+Z_{s_{n}}\right|^{2}}\right] \mathrm{d} s_{1} \ldots \mathrm{d} s_{n} \\
= & n ! \int_{0 \leqslant s_{1} \leqslant \cdots \leqslant s_{n-1} \leqslant t}^{t} E_{0}\left[\frac{1}{\left|u+Z_{s_{1}}\right|^{2}} \cdots \frac{1}{\left|u+Z_{s_{n-1}}\right|^{2}}\right. \\
& \left.\times \int_{s_{n-1}}^{t} E_{Z_{s_{n-1}}}\left[\frac{1}{\left|u+Z_{s_{n}-s_{n-1}}\right|^{2}}\right] \mathrm{d} s_{n}\right] \mathrm{d} s_{1} \ldots \mathrm{d} s_{n-1} .
\end{aligned}
$$


From the estimate (25), the previous expression can be bounded by

$$
n ! c_{T}^{\prime} \int_{0 \leqslant s_{1} \leqslant \cdots \leqslant s_{n-1} \leqslant t} E_{0}\left[\frac{1}{\left|u+Z_{s_{1}}\right|^{2}} \cdots \frac{\left(1+\log _{+} \frac{1}{\mid u+Z_{s_{n-1} \mid}}\right)}{\left|u+Z_{s_{n-1}}\right|^{2}}\right] \mathrm{d} s_{1} \ldots \mathrm{d} s_{n-1} .
$$

By induction we estimate this expression by (recall that $u=x-y$ )

$$
n !\left(c_{T}^{\prime}\right)^{n}\left(1+\log _{+}^{n} \frac{1}{|x-y|}\right) .
$$

We have completed the proof that the moments are finite. All the claims of the theorem concerning $I_{1}(x-y)$ are proved.

Remark 7. - For $x=y$, one has

$$
\int_{0}^{t} \frac{1}{\left|W_{t}-W_{s}\right|^{2}} \mathrm{~d} s=+\infty \quad P \text {-a.s. }
$$

see [25, p. 9]. So $x=y$ in (26) is not admissible even without taking the expectation.

Step 2. Let us recall some well-known facts about $\alpha(x-y ; T)$ needed to prove the theorem. The existence of a continuous field $\alpha(u ; T), u \neq 0$, called intersection local time can be found in Rosen [20,21], Le Gall [14] (with the easiest proof of continuity), Yor [29]. In fact $\alpha(u ; T)$ has Hölder continuity properties that can be found in these references. In Yor [29] the representation

$$
\begin{aligned}
2 \pi \alpha(u ; T)= & \int_{0}^{t}\left(\frac{1}{|u|}-\frac{1}{\left|u+W_{s}-W_{t}\right|}\right) \mathrm{d} s \\
& +\int_{0}^{t}\left(\int_{0}^{s} \frac{u+W_{r}-W_{s}}{\left|u+W_{r}-W_{s}\right|^{3}} \mathrm{~d} r\right) \mathrm{d} W_{s}
\end{aligned}
$$

is proved (similar to the one of Rosen [21]) which easily implies that the moments of all orders are finite: this property for the first integral is recalled in step 3 below, while for the stochastic integral we have

$$
\begin{gathered}
E\left[\sup _{t \in[0, T]}\left|\int_{0}^{t}\left(\int_{0}^{s} \frac{u+W_{r}-W_{s}}{\left|u+W_{r}-W_{s}\right|^{3}} \mathrm{~d} r\right) \mathrm{d} W_{s}\right|^{2 n}\right] \\
\leqslant C_{n, T} \int_{0}^{T} E\left[\left.|| \int_{0}^{s} \frac{u+W_{r}-W_{s}}{\left|u+W_{r}-W_{s}\right|^{3}} \mathrm{~d} r\right|^{2 n}\right] \mathrm{d} s \\
\leqslant C_{n, T} \int_{0}^{T} E\left[\left|\int_{0}^{s} \frac{1}{\left|u+W_{r}-W_{s}\right|^{2}} \mathrm{~d} r\right|^{2 n}\right] \mathrm{d} s
\end{gathered}
$$

which is finite by step 1 above. These are the properties needed to prove the theorem. 
Step 3. Finally, we have to deal with $I_{4}(x-y)$. It is clear that it is a measurable field with finite moments, since it is bounded above by the similar integrals of step 1 . However, the computations of Khasminskii lemma in this case readily give us

$$
E\left[I_{4}(x-y)^{2 n}\right] \leqslant(2 n) !\left(c_{T}^{\prime \prime}\right)^{2 n}
$$

for some constant $c_{T}^{\prime \prime}>0$. It has also a continuous version, in fact Hölder continuous with any exponent $\alpha<1$, with $x-y$ varying over the whole $\mathbf{R}^{3}$, as it is proved by Rosen [21, Lemma 1]. This completes the proof of the theorem.

Remark 8. - We do not know whether $I_{1}(x-y)$ has a continuous version for $x \neq y$; and in particular we cannot say whether $H_{x y}(\omega)$ is finite for all $x \neq y$, for $P$-a.e. given $\omega \in \Omega$. An obvious attempt to apply the classical Kolmogorov criterium lead us to the estimate

$$
E\left[\left|I_{1}(u)-I_{1}(v)\right|^{2 n}\right] \leqslant C_{n, T}^{\prime} \int_{0}^{T} E\left[\left(\int_{0}^{t}\left(\frac{1}{\left|u+W_{t}-W_{s}\right|}-\frac{1}{\left|v+W_{t}-W_{s}\right|}\right)^{2} \mathrm{~d} s\right)^{n}\right] \mathrm{d} t .
$$

Using a bound employed by Rosen [21] we end up to estimate the term

$$
\leqslant C_{n, T}^{\prime \prime}|u-v|^{2 n \alpha} \int_{0}^{T} E\left[\left(\int_{0}^{t} \frac{1}{\left|u+W_{t}-W_{s}\right|^{2+2 \alpha}} \mathrm{d} s\right)^{n}\right] \mathrm{d} t
$$

and the similar one with $v$ in place of $u$. But now the iterative procedure, similar to Khasminskii lemma, recalled in step 2 above fails, since at every step we increase the singularity by $2 \alpha$, and we cannot pass the threshold $2+2 n \alpha<3$ (otherwise we get divergent integrals also for $u \neq 0$, see the computations of step 1 in the proof of Theorem 6). This implies $2 n \alpha<1$, in contrast to the requirement $2 n \alpha>1$ necessary to apply Kolmogorov regularity theorem (at least radially). Similarly, we have not been able to adapt the interesting approach of Le Gall [14] to the continuity of $I_{1}$.

Next theorem describes the scaling properties of the interaction energy when $\mid x-$ $y \mid \rightarrow 0$. Essentially, the result says that $H_{x y}$ behaves like $\frac{1}{|x-y|}$, up to lower order terms (just logaritmic corrections). This is the origin of the condition (2) to have a finite energy $H$.

THEOREM 9. - There exists a constant $C_{n, T}>0$ such that

$$
E\left[\left(H_{x y}-\frac{\Gamma^{2}}{8 \pi} \frac{2 T}{|x-y|}\right)^{2 n}\right]^{\frac{1}{2 n}} \leqslant C_{n, T}\left(1+\log _{+} \frac{1}{|x-y|}\right)
$$

for all $x \neq y$. The dominating term in $H_{x y}^{\left(\frac{1}{2}\right)}\left(\right.$ similarly for $\left.H_{y x}^{\left(\frac{1}{2}\right)}\right)$ is

$$
\frac{\Gamma^{2}}{8 \pi}\left(\pi \alpha(x-y ; T)+\frac{1}{2} \frac{T}{|x-y|}\right)
$$


which behaves as $\frac{\Gamma^{2}}{8 \pi} \frac{T}{|x-y|}$ as $|x-y| \rightarrow 0$, while the other terms have a lower order; we understand these statements in the following sense:

$$
\begin{gathered}
E\left[I_{1}(x-y)^{2 n}\right]^{\frac{1}{2 n}} \leqslant C_{n, T}^{\prime}\left(1+\log _{+} \frac{1}{|x-y|}\right), \\
E\left[I_{4}(x-y)^{2 n}\right]^{\frac{1}{2 n}} \leqslant((2 n) !)^{\frac{1}{2 n}} C_{T},
\end{gathered}
$$

$\left(C_{n, T}, C_{n, T}^{\prime}\right.$ and $C_{T}$ are suitable constants depending on the indicated arguments; see also Remark 12 for $C_{n, T}^{\prime}$ )

$$
\frac{1}{\sqrt{\log \frac{1}{|x-y|}}}\left\{\alpha(x-y ; T)-\frac{T}{2 \pi|x-y|}\right\} \rightarrow 2 B_{T}
$$

as $|x-y| \rightarrow 0$, where $B_{T}$ is a standard Brownian motion at time $T$, and the convergence is in law.

Remark 10. - Imkeller et al. [11] prove that the convergence (33) holds true in the weak sense of $D^{2, \alpha}$ for any $\alpha<\frac{1}{2}$, where $D^{2, \alpha}$ is the Sobolev space of order $\alpha$ over the Wiener space.

Proof. - Properties (31) and (32) have been proved above, see (24), (27) and (29). From (28), (31) and (32) we have (by the same computations following (28))

$$
E\left[\left(2 \pi \alpha(x-y ; T)-\frac{T}{|x-y|}\right)^{2 n}\right]^{\frac{1}{2 n}} \leqslant C_{n, T}^{\prime \prime}\left(1+\log _{+} \frac{1}{|x-y|}\right)
$$

for some constant $C_{n, T}^{\prime \prime}$. Now (30) follows from (31), (32) and (34). Finally, (33) has been proved by Yor [30].

THEOREM 11. - Under the condition (2), the kynetic energy $H$ of the vortex structure $\left(C_{\mathcal{A}}, \rho\right)$ is a well defined real valued random variable, with finite moments of every order.

Proof. - Since $H_{x y}$ is a measurable random field (i.e., jointly measurable in $(x, y, \omega)$ ), the double integral

$$
\int_{\mathcal{A}} \int_{\mathcal{A}}\left|H_{x y}\right| \rho(\mathrm{d} x) \rho(\mathrm{d} y)
$$

is well defined $P$-a.s., maybe infinite, and it is measurable in $\omega$. We have

$$
E \int_{\mathcal{A}} \int_{\mathcal{A}}\left|H_{x y}\right| \rho(\mathrm{d} x) \rho(\mathrm{d} y)=\int_{\mathcal{A}} \int_{\mathcal{A}} E\left|H_{x y}\right| \rho(\mathrm{d} x) \rho(\mathrm{d} y) \leqslant \int_{\mathcal{A}} \int_{\mathcal{A}} \frac{C}{|x-y|} \rho(\mathrm{d} x) \rho(\mathrm{d} y)<\infty
$$

by the result of the previous theorem and assumption (2). Therefore

$$
\int_{\mathcal{A}} \int_{\mathcal{A}}\left|H_{x y}\right| \rho(\mathrm{d} x) \rho(\mathrm{d} y)<\infty \quad P \text {-a.s. }
$$


which in turn implies that $\int_{\mathcal{A}} \int_{\mathcal{A}} H_{x y} \rho(\mathrm{d} x) \rho(\mathrm{d} y)$ is well defined and finite $P$-a.s.

Concerning the moments, they are finite since $\rho$ is a probability measure and $H_{x y}$ has property (30):

$$
\begin{aligned}
& E\left[\left|\int_{\mathcal{A}} \int_{\mathcal{A}} H_{x y} \rho(\mathrm{d} x) \rho(\mathrm{d} y)\right|^{2 n}\right] \\
& \quad \leqslant C_{n} E\left[\left|\int_{\mathcal{A}} \int_{\mathcal{A}}\left(H_{x y}-\frac{\Gamma^{2}}{8 \pi} \frac{2}{|x-y|}\right) \rho(\mathrm{d} x) \rho(\mathrm{d} y)\right|^{2 n}\right]+C_{n}^{\prime} \\
& \leqslant C_{n} \int_{\mathcal{A}} \int_{\mathcal{A}} E\left[\left(H_{x y}-\frac{\Gamma^{2}}{8 \pi} \frac{2}{|x-y|}\right)^{2 n}\right] \rho(\mathrm{d} x) \rho(\mathrm{d} y)+C_{n}^{\prime} \\
& \leqslant C_{n}^{\prime \prime} \int_{\mathcal{A}} \int_{\mathcal{A}}\left(1+\log _{+}^{2 n} \frac{1}{|x-y|}\right) \rho(\mathrm{d} x) \rho(\mathrm{d} y)+C_{n}^{\prime}<\infty .
\end{aligned}
$$

The proof is complete.

Remark 12. - Unfortunately, the estimates on the moments given in the proof of Theorem 6 give us only $E\left[|H|^{n}\right] \leqslant C^{n} n^{2 n}$ for some $C>0$. Therefore it is not clear if $E\left[\mathrm{e}^{\lambda H}\right]$ is finite, at least for small $|\lambda|$. In view of Gibbs measures, one is interested in $\lambda=-\beta$, first with $\beta>0$, but also with (at least small) $\beta<0$ in the framework of vortex structures. Even for positive inverse temperature $\beta$ we do not have a final answer, see Remark 1, but a work in progress seems to give us hope.

\section{Acknowledgement}

I express my gratitude to A. Chorin, P. Imkeller, F. Le Gall, and M. Barlow for some important comments, to $\mathrm{M}$. Isopi for a carefull reading of the manuscript and many advices, and to my collaborators M. Barsanti, L. Berselli, H. Bessaih, B. Busnello, M. Gubinelli, F. Morandin, M. Romito for the help deriving from common efforts in related research directions. The research has been partially supported by the MURST project on Stochastic Processes with Spatial Structure.

\section{REFERENCES}

[1] Akao J., Unpublished manuscript, UC Berkeley, 1996; Phase transitions and connectivity in the three-dimensional vortex equilibria, Ph.D Thesis, UC Berkeley, 1994.

[2] Albeverio S., Cruzeiro A.B., Global flows with invariant (Gibbs) measures for Euler and Navier-Stokes two-dimensional fluids, Comm. Math. Phys. 129 (1990) 431-444.

[3] Bell J., Marcus D., Vorticity intensification and the transition to turbulence in the threedimensional Euler equations, Comm. Math. Phys. 147 (1992) 371-394.

[4] Bolthausen E., On the construction of the three-dimensional polymer measure, Probab. Theory Related Fields 97 (1993) 81-101.

[5] Chorin A., Vorticity and Turbulence, Springer, New York, 1994.

[6] Crauel H., Flandoli F., Attractors for random dynamical systems, Probab. Theory Related Fields 100 (1994) 365-393. 
[7] Flandoli F., Dissipativity and invariant measures for stochastic Navier-Stokes equations, Nonlin. Diff. Eq. Appl. 1 (1994) 403-423.

[8] Flandoli F., Ga̧tarek D., Martingale and stationary solutions for stochastic Navier-Stokes equations, Probab. Theory Related Fields 102 (1995) 367-391.

[9] Flandoli F., Maslowski B., Ergodicity of the 2-D Navier-Stokes equation under random perturbations, Comm. Math. Phys. 171 (1995) 119-141.

[10] Gallavotti G., Meccanica dei Fluidi, Quaderni CNR - GNAFA, Vol. 52, Roma, 1996.

[11] Imkeller P., Perez-Abreu V., Vives J., Chaos expansions of double intersection local time of Brownian motion in $\mathbf{R}^{d}$ and renormalization, Stoch. Proc. Their Appl. 56 (1995) 1-34.

[12] Kunita H., Stochastic differential equations and stochastic flows of diffeomorphisms, in: Hennequin P.L. (Ed.), École d'été de Saint-Flour XII, 1982, LNM, Vol. 1097, Springer, Berlin, 1984.

[13] Landkof N.S., Foundations of Modern Potential Theory, Springer, New York, 1972.

[14] Le Gall J.F., Sur le temps local d'intersection du mouvement Brownien plan, et la méthode de renormalisation de Varadhan, in: Sém. de Prob. XIX 1983/84, LNM, Vol. 1123, Springer, Berlin, 1985, pp. 314-331.

[15] Le Gall J.F., Some properties of planar Brownian motion, in: École d'été de Saint-Flour XX, 1990, LNM, Vol. 1527, Springer, Berlin, 1992.

[16] Lions P.L., On Euler Equations and Statistical Physics, Quaderni Scuola Nornale Superiore of Pisa, Pisa, 1997.

[17] Marchioro C., Pulvirenti M., Mathematical Theory of Incompressible Nonviscous Fluids, Springer, Berlin, 1994.

[18] Lions P.L., Majda A., Equilibrium statistical theory for nearly parallel vortex filaments, Comm. Pure Appl. Math. 53 (2000), to appear.

[19] Revuz D., Yor M., Continuous Martingales and Brownian Motion, Springer, Berlin, 1994.

[20] Rosen J., A local time approach to the self-intersections of Brownian paths in space, Comm. Math. Phys. 88 (1983) 327-338.

[21] Rosen J., A representation for the intersection local time of Brownian motion in space, Ann. Probab. 13 (1985) 145-153.

[22] Ruiz de Chavez J., Sur les integrales stochastiques multiples, in: Sém. de Prob. XIX, 1983/84, LNM, Vol. 1123, Springer, Berlin, 1985, pp. 248-262.

[23] Russo F., Vallois P., The generalized covariation process and Ito formula, Stoch. Proc. Their Appl. 59 (1995) 81-104.

[24] Russo F., Vallois P., Ito formula for $C^{1}$-functions of semimartinagales, Probab. Theory Relat. Fields 104 (1996) 27-41.

[25] Sznitman A.-S., Brownian Motion, Obstacles and Random Media, Springer, Berlin, 1998.

[26] Toth B., Werner W., The true self-repelling motion, Probab. Theory Related Fields 111 (1998) 375-452.

[27] Vishik M.I., Fursikov A.V., Mathematical Problems of Statistical Hydromechanics, Kluwer, Dordrecht, 1980.

[28] Westwater J., On Edwards model for long polymer chains, Comm. Math. Phys. 72 (1980) 131-174.

[29] Yor M., Compléments aux formules de Tanaka-Rosen, in: Sém. de Prob. XIX, 1983/84, LNM, Vol. 1123, Springer, Berlin, 1985, pp. 332-348.

[30] Yor M., Renormalisation et convergence en loi pour le temps locaux d'intersection du mouvement brownien dans $\mathbf{R}^{3}$, in: Sém. de Prob. XIX, 1983/84, LNM, Vol. 1123, Springer, Berlin, 1985, pp. 350-365. 